\title{
Bifurcation Analysis of a Delayed Worm Propagation Model with Saturated Incidence
}

\author{
Zizhen Zhang $\mathbb{D},{ }^{1}$ Yougang Wang, ${ }^{1}$ and Luca Guerrini $\mathbb{D}^{2}$ \\ ${ }^{1}$ School of Management Science and Engineering, Anhui University of Finance and Economics, Bengbu 233030, China \\ ${ }^{2}$ Department of Management, Marche Polytechnic University, Piazza Martelli 8, 60121 Ancona, Italy
}

Correspondence should be addressed to Zizhen Zhang; zzzhaida@163.com

Received 2 March 2018; Accepted 17 April 2018; Published 15 May 2018

Academic Editor: Ming Mei

Copyright (c) 2018 Zizhen Zhang et al. This is an open access article distributed under the Creative Commons Attribution License, which permits unrestricted use, distribution, and reproduction in any medium, provided the original work is properly cited.

This paper is concerned with a delayed SVEIR worm propagation model with saturated incidence. The main objective is to investigate the effect of the time delay on the model. Sufficient conditions for local stability of the positive equilibrium and existence of a Hopf bifurcation are obtained by choosing the time delay as the bifurcation parameter. Particularly, explicit formulas determining direction of the Hopf bifurcation and stability of the bifurcating periodic solutions are derived by using the normal form theory and the center manifold theorem. Numerical simulations for a set of parameter values are carried out to illustrate the analytical results.

\section{Introduction}

Worms, as one kind of malicious codes, have become one of the main threats to the security of networks. Since the first Morris worm in 1998, new worms have come into networks frequently, including Slammer worm [1], Commwarrior worm [2], Cabir worm [3], and Chameleon worm [4]. Each of them can cause enormous financial losses and social panic [5-7]. Therefore, it is significant to explore effective methods to counter against worms. To this end, we need to accurately understand the dynamic behaviors of worm propagation in networks. Considering that the process of worm propagation in networks is similar to that of biological virus propagation in the population, mathematical models have been important tools used to analyze the propagation and control of worms based on the theory of Kermack and McKendrick [8].

In [9], Kim et al. proposed the SIS (SusceptibleInfectious-Susceptible) model in order to analyze the dynamical behaviors of worm propagation on Internet. However, the SIS model neglects the effect of the antivirus software. Thus, the SIR (Susceptible-Infectious-Recovered) model is proposed [9]. Although SIR model considered the immunity of the nodes in which the worms have been cleaned, however, it assumes that the recovered hosts have permanent immunity. This is not consistent with the reality in networks, because they may be infected by some new emerging worms again. To overcome this drawback of the SIR model, Wang et al. investigated the SIRS (Susceptible-Infectious-RecoveredSusceptible) mode for analyzing the dynamics of worm propagation in networks [10-12]. It should be pointed out that both the SIR mode and the SIRS model assume that the susceptible nodes become infectious instantaneously. As we know, worms usually have a latent period. Based on this consideration, the SEIR (Susceptible-Exposed-InfectiousRecovered) model $[13,14]$ and the SEIRS (SusceptibleExposed-Infectious-Recovered-Susceptible) model [11, 15] are proposed to describe the dynamics of worm propagation in networks. Considering influence of the quarantine strategy and the vaccination strategy on the propagation of worms, some worm models with quarantine strategy [16-19] and vaccination strategy [20-25] are formulated and analyzed.

It should be pointed out that all the models above use the bilinear incidence rate $\beta S I$. As stated in [26], the dynamics of a model system heavily depends on the choice of the incidence rate. Gan et al. have considered the different incidence rate functions $\beta S I / f(I)$ in their work $[27,28]$. It was found that the saturated incidence rate $\beta S I /(1+\eta I)$ is more general than the bilinear incidence rate $\beta S I$. Based on this, 
Wang et al. [29] proposed the following model with partial immunization to defend against worms:

$$
\begin{aligned}
& \frac{d S(t)}{d t}=(1-p) A-\frac{\beta S(t) I(t)}{1+\eta I(t)}-\mu S(t)+\gamma V(t), \\
& \frac{d V(t)}{d t}=p A-\sigma \beta V(t) I(t)-(\mu+\gamma) V(t), \\
& \frac{d E(t)}{d t}=\frac{\beta S(t) I(t)}{1+\eta I(t)}+\sigma \beta V(t) I(t)-(\mu+\omega) E(t), \\
& \frac{d I(t)}{d t}=\omega E(t)-(\mu+\alpha+\delta) I(t), \\
& \frac{d R(t)}{d t}=\delta I(t)-\mu R(t),
\end{aligned}
$$

where $S(t), V(t), E(t), I(t)$, and $R(t)$ present numbers of the susceptible, vaccinated, exposed, infectious, recovered hosts at time $t$, respectively. The meanings of more parameters are described and shown in "Parameters of the Model and Their Meanings" section. Wang et al. [29] investigated the stability of system (1).

One of the significant features of computer viruses is their latent characteristics $[30,31]$. In addition, time delays of one type or another could cause the numbers of hosts in system (1) to fluctuate. And worm propagation models with time delay have been investigated by some scholars [14, 17, 19]. Based on above discussions, in this paper, we extend system (1) by incorporating the time delay due to the latent period of the worms in the exposed hosts into system (1) and obtain the following delayed worm propagation model:

$$
\begin{aligned}
\frac{d S(t)}{d t}= & (1-p) A-\frac{\beta S(t) I(t)}{1+\eta I(t)}-\mu S(t)+\gamma V(t), \\
\frac{d V(t)}{d t}= & p A-\sigma \beta V(t) I(t)-(\mu+\gamma) V(t), \\
\frac{d E(t)}{d t}= & \frac{\beta S(t) I(t)}{1+\eta I(t)}+\sigma \beta V(t) I(t)-\mu E(t) \\
& -\omega E(t-\tau), \\
\frac{d I(t)}{d t}= & \omega E(t-\tau)-(\mu+\alpha+\delta) I(t), \\
\frac{d R(t)}{d t}= & \delta I(t)-\mu R(t),
\end{aligned}
$$

where $\tau$ is the latent period of the worms in the exposed nodes.

The remainder of this paper is organized as follows. Local stability of the positive equilibrium and existence of a Hopf bifurcation at the positive equilibrium are analyzed in the next section. Properties of the Hopf bifurcation such as direction and stability are investigated in Section 3. Numerical simulations are carried out in Section 4 to support the obtained theoretical results. Finally, conclusions are given in Section 5 to end our work.

\section{Existence of Hopf Bifurcation}

By direct computation, we know that if the condition $\left(H_{1}\right)$ : $(\mu+\omega)(\mu+\alpha+\delta)\left(\sigma \beta I_{*}+\mu+\gamma\right)>\omega \sigma \beta p A$ holds, then system (2) has a positive equilibrium $P_{*}\left(S_{*}, V_{*}, E_{*}, I_{*}, R_{*}\right)$, where

$S_{*}$

$$
\begin{aligned}
& =\frac{\left(1+\eta I_{*}\right)\left[(\mu+\omega)(\mu+\alpha+\delta)\left(\sigma \beta I_{*}+\mu+\gamma\right)-\omega \sigma \beta p A\right]}{\beta \omega\left(\sigma \beta I_{*}+\mu+\gamma\right)}, \\
& V_{*}=\frac{p A}{\sigma \beta I_{*}+\mu+\gamma}, \\
& E_{*}=\frac{\mu+\alpha+\delta}{\omega} I_{*}, \\
& R_{*}=\frac{\delta}{\mu} I_{*} .
\end{aligned}
$$

And $I_{*}$ is the positive root of the following equation:

$$
P_{2} x^{2}+P_{1} x+P_{0}=0,
$$

where

$$
\begin{aligned}
P_{0}= & \mu(\mu+\omega)(\mu+\gamma)(\mu+\alpha+\delta) \\
& -A \omega \beta(\gamma+p \mu \delta+(1-p) \mu), \\
P_{1}= & (\mu+\omega)(\mu+\alpha+\delta)[\sigma \beta \mu+(\mu+\gamma)(\mu \eta+\beta)] \\
& -A \omega \sigma \beta(\beta+p \mu \eta), \\
P_{2}= & \beta \sigma(\mu+\omega)(\mu+\alpha+\delta)(\beta+\mu \eta) .
\end{aligned}
$$

The Jacobi matrix of system (2) about $P_{*}\left(S_{*}, V_{*}, E_{*}, I_{*}, R_{*}\right)$ is given by

$$
J\left(P_{*}\right)=\left(\begin{array}{ccccc}
m_{11} & m_{12} & 0 & m_{14} & 0 \\
0 & m_{22} & 0 & m_{24} & 0 \\
m_{31} & m_{32} & m_{33}+n_{33} e^{-\lambda \tau} & m_{34} & 0 \\
0 & 0 & n_{43} e^{-\lambda \tau} & m_{44} & 0 \\
0 & 0 & 0 & m_{54} & m_{55}
\end{array}\right),
$$

where

$$
\begin{aligned}
& m_{11}=-\left(\frac{\beta I_{*}}{1+\eta I_{*}}+\mu\right), \\
& m_{12}=\gamma, \\
& m_{14}=-\frac{\beta S_{*}}{\left(1+\eta I_{*}\right)^{2}}, \\
& m_{22}=-\left(\mu+\gamma+\sigma \beta I_{*}\right), \\
& m_{24}=-\sigma \beta V_{*}, \\
& m_{31}=\frac{\beta I_{*}}{1+\eta I_{*}}, \\
& m_{32}=\sigma \beta I_{*}, \\
& m_{33}=-\mu,
\end{aligned}
$$




$$
\begin{aligned}
& m_{34}=\frac{\beta S_{*}}{\left(1+\eta I_{*}\right)^{2}}+\sigma \beta V_{*}, \\
& m_{44}=-(\mu+\alpha+\delta), \\
& m_{54}=\delta \\
& m_{55}=-\mu, \\
& n_{33}=-\omega, \\
& n_{43}=\omega .
\end{aligned}
$$

The characteristic equation of that matrix (6) is

$$
\begin{aligned}
\lambda^{5}+ & M_{4} \lambda^{4}+M_{3} \lambda^{3}+M_{2} \lambda^{2}+M_{1} \lambda+M_{0} \\
& +\left(N_{4} \lambda^{4}+N_{3} \lambda^{3}+N_{2} \lambda^{2}+N_{1} \lambda+N_{0}\right) e^{-\lambda \tau}=0
\end{aligned}
$$

with

$$
\begin{aligned}
M_{0} & =-m_{11} m_{22} m_{33} m_{44} m_{55}, \\
M_{1} & =m_{11} m_{22} m_{33} m_{44}+m_{55}\left[m_{11} m_{22}\left(m_{33}+m_{44}\right)\right. \\
& \left.+m_{33} m_{44}\left(m_{11}+m_{22}\right)\right] \\
M_{2} & =-\left[m_{11} m_{22}\left(m_{33}+m_{44}\right)+m_{33} m_{44}\left(m_{11}+m_{22}\right)\right] \\
& -m_{55}\left[m_{11} m_{22}+m_{33} m_{44}\right. \\
& \left.+\left(m_{11}+m_{22}\right)\left(m_{33}+m_{44}\right)\right] \\
M_{3} & =m_{11} m_{22}+m_{33} m_{44}+\left(m_{11}+m_{22}\right)\left(m_{33}+m_{44}\right) \\
& +m_{55}\left(m_{11}+m_{22}+m_{33}+m_{44}\right) \\
M_{4} & =-\left(m_{11}+m_{22}+m_{33}+m_{44}+m_{55}\right) \\
& \left.+\left(m_{11}+m_{22}\right)\left(m_{44}+m_{55}\right)\right], \\
N_{0} & =m_{11} m_{22} m_{55} n_{43}\left(m_{34}-m_{44}\right) \\
& +m_{55} n_{43}\left(m_{14} m_{22} m_{31}-m_{11} m_{24} m_{32}\right. \\
& +n_{4} \\
& \left.-m_{12} m_{24} m_{31}\right), \\
& \left.+m_{11} m_{55}+m_{11}+m_{22}+m_{44}+m_{55}-m_{34}\right) \\
N_{2} & =n_{43}\left(m_{14} m_{31}-m_{24} m_{32}\right)+m_{34} n_{43}\left(m_{11}+m_{22}\right. \\
N_{1} & =n_{43}\left[m_{11} m_{22}\left(m_{44}+m_{55}\right)+m_{44} m_{55}\left(m_{11}+m_{22}\right)\right. \\
& \left.+m_{31}\right]+\left[m_{24} m_{32} n_{43}\left(m_{11}+m_{55}\right)\right]-m_{34} n_{43}\left(m_{11} m_{22}\right.
\end{aligned}
$$

When $\tau=0$, (8) becomes

$$
\lambda^{5}+M_{04} \lambda^{4}+M_{03} \lambda^{3}+M_{02} \lambda^{2}+M_{01} \lambda+M_{00}=0,
$$

where

$$
\begin{aligned}
& M_{00}=M_{0}+N_{0}, \\
& M_{01}=M_{1}+N_{1}, \\
& M_{02}=M_{2}+N_{2}, \\
& M_{03}=M_{3}+N_{3}, \\
& M_{04}=M_{4}+N_{4} .
\end{aligned}
$$

Thus, $P_{*}\left(S_{*}, E_{*}, I_{*}, R_{*}, V_{*}\right)$ is locally asymptotically stable when $\tau=0$ if the condition $\left(\mathrm{H}_{2}\right)$ is satisfied and $\left(\mathrm{H}_{2}\right)$ is defined as follows:

$$
\begin{aligned}
& M_{00}>0 \\
& M_{04}>0 \\
& M_{03} M_{04}>M_{02}, \\
& M_{02}\left(M_{01}+M_{03} M_{04}\right)>M_{01} M_{04}^{2}+M_{02}^{2}, \\
& M_{02} M_{03}\left(M_{00}+M_{01}+M_{02}\right)+2 M_{00} M_{01} M_{04} \\
& \quad>M_{00}^{2}+M_{01} M_{02}^{2}+M_{04}\left(M_{01}^{2} M_{04}+M_{00} M_{03}^{2}\right) .
\end{aligned}
$$

For $\tau>0$, let $\lambda=i \omega(\omega>0)$ be the root of (8). Then, we have

$$
\begin{aligned}
& \left(N_{1} \omega-N_{3} \omega^{3}\right) \sin \tau \omega+\left(N_{4} \omega^{4}-N_{2} \omega^{2}+N_{0}\right) \cos \tau \omega \\
& \quad=M_{2} \omega^{2}-M_{4} \omega^{4}-M_{0}, \\
& \left(N_{1} \omega-N_{3} \omega^{3}\right) \cos \tau \omega-\left(N_{4} \omega^{4}-N_{2} \omega^{2}+N_{0}\right) \sin \tau \omega \\
& \quad=M_{3} \omega^{3}-\omega^{5}-M_{1} \omega .
\end{aligned}
$$

Thus, we can get the following equation:

$$
\omega^{10}+h_{4} \omega^{8}+h_{3} \omega^{6}+h_{2} \omega^{4}+h_{1} \omega^{2}+h_{0}=0,
$$

where

$$
\begin{aligned}
h_{0}= & M_{0}^{2}-N_{0}^{2}, \\
h_{1}= & M_{1}^{2}-2 M_{0} M_{2}-N_{1}^{2}+2 N_{0} N_{2}, \\
h_{2}= & M_{2}^{2}+2 M_{0} M_{4}-2 M_{1} M_{3}+2 N_{1} N_{3}-N_{2}^{2} \\
& -2 N_{0} N_{4}, \\
h_{3}= & M_{3}^{2}-2 M_{2} M_{4}+2 M_{1}-N_{3}^{2}+2 N_{2} N_{4}, \\
h_{4}= & M_{4}^{2}-2 M_{3}-N_{4}^{2} .
\end{aligned}
$$

Let $v=\omega^{2}$; then (14) becomes

$$
v^{5}+h_{4} v^{4}+h_{3} v^{3}+h_{2} \omega^{2}+h_{1} v+h_{0}=0 .
$$

Based on the discussion about the distribution of the roots of (16) in [32], we suppose that $\left(H_{3}\right)$ : (16) has at least one positive root $v_{0}$. 
If the condition $\left(\mathrm{H}_{3}\right)$ holds, then (16) has a positive root $\omega_{0}=\sqrt{v_{0}}$ and (8) has a pair of purely imaginary roots $\pm i \omega_{0}$. For $\omega_{0}$, we have

$$
\tau_{0}=\frac{1}{\omega_{0}} \times \arccos \left\{\frac{G_{1}(\omega)}{G_{2}(\omega)}\right\},
$$

with

$$
\begin{aligned}
G_{1}(\omega) & \\
= & \left(N_{3}-M_{4} N_{4}\right) \omega^{8} \\
& +\left(M_{2} N_{4}+M_{4} N_{2}-M_{3} N_{3}-N_{1}\right) \omega^{6} \\
& +\left(M_{3} N_{1}+M_{1} N_{3}-M_{0} N_{4}-M_{2} N_{2}-M_{4} N_{0}\right) \omega^{4} \\
& +\left(M_{0} N_{2}+M_{2} N_{0}-M_{1} N_{1}\right) \omega^{2}+M_{0} N_{0}, \\
G_{2}(\omega) & \\
= & N_{4}^{2} \omega^{8}+\left(N_{3}^{2}-2 N_{2} N_{4}\right) \omega^{6} \\
& +\left(N_{2}^{2}+2 N_{0} N_{4}-2 N-1 N_{3}\right) \omega^{4} \\
& +\left(N_{1}^{2}-2 N_{0} N_{2}\right) \omega^{2}+N_{0}^{2} .
\end{aligned}
$$

Differentiating on both sides of (8) with respect to $\tau$, we can obtain

$$
\begin{aligned}
& {\left[\frac{d \lambda}{d \tau}\right]^{-1}} \\
& =\frac{\left(5 \lambda^{4}+4 M_{4} \lambda^{3}+3 M_{3} \lambda^{2}+2 M_{2} \lambda+M_{1}\right) e^{\lambda \tau}}{\lambda\left(N_{4} \lambda^{4}+N_{3} \lambda^{3}+N_{2} \lambda^{2}+N_{1} \lambda+N_{0}\right)} \\
& \quad-\frac{\tau}{\lambda}
\end{aligned}
$$

Further, we have

$$
\operatorname{Re}\left[\frac{d \lambda}{d \tau}\right]_{\tau=\tau_{0}}^{-1}=\frac{f^{\prime}\left(v_{0}\right)}{G_{2}\left(\omega_{0}\right)}
$$

where $f(v)=v^{5}+h_{4} v^{4}+h_{3} v^{3}+h_{2} \omega^{2}+h_{1} v+h_{0}$

Obviously, if the condition $\left(H_{4}\right): f^{\prime}\left(v_{0}\right) \neq 0$ is satisfied, then $\operatorname{Re}[d \lambda / d \tau]_{\tau=\tau_{0}}^{-1} \neq 0$. Based on the discussion above and the Hopf bifurcation theorem in [33], we have the following results.

Theorem 1. For system (2), if the conditions $\left(H_{1}\right)-\left(H_{4}\right)$ hold, then the positive equilibrium $P_{*}\left(S_{*}, V_{*}, E_{*}, I_{*}, R_{*}\right)$ is locally asymptotically stable when $\tau \in\left[0, \tau_{0}\right)$; system (2) undergoes a Hopf bifurcation at the $P_{*}\left(S_{*}, V_{*}, E_{*}, I_{*}, R_{*}\right)$ when $\tau=\tau_{0}$ and a family of periodic solutions bifurcate from $P_{*}\left(S_{*}, V_{*}, E_{*}, I_{*}, R_{*}\right)$.

\section{Properties of the Hopf Bifurcation}

Let $u_{1}(t)=S(t)-S_{*}, u_{2}(t)=V(t)-V_{*}, u_{3}(t)=E(t)-E_{*}$, $u_{4}(t)=I(t)-I_{*}$, and $u_{5}(t)=R(t)-R_{*}$, and normalize the time delay with the scaling $t \rightarrow(t / \tau)$. Let $\tau=\tau_{0}+$ $\varrho(\varrho \in R)$; then $\varrho=0$ is the Hopf bifurcation value of system (2). System (2) can be transformed into the following form:

$$
\dot{u}(t)=L_{\varrho}\left(u_{t}\right)+F\left(\varrho, u_{t}\right)
$$

where $u(t)=\left(u_{1}, u_{2}, u_{3}, u_{4}, u_{5}\right)^{T} \in C=C\left([-1,0], R^{5}\right)$ and $L_{\varrho}: C \rightarrow R^{5}$ and $F: R \times C \rightarrow R^{5}$ are given, respectively, by

$$
\begin{aligned}
L_{\varrho} \phi & =\left(\tau_{0}+\varrho\right)\left(M_{1} \phi(0)+M_{2} \phi(-1)\right), \\
& F(\varrho, \phi)=\left(\begin{array}{c}
m_{15} \phi_{1}(0) \phi_{4}(0)+m_{16} \phi_{4}^{2}(0)+m_{17} \phi_{1}(0) \phi_{4}^{2}(0)+m_{18} \phi_{4}^{3}(0)+\cdots \\
m_{25} \phi_{2}(0) \phi_{4}(0) \\
m_{35} \phi_{1}(0) \phi_{4}(0)+m_{36} \phi_{4}^{2}(0)+m_{37} \phi_{1}(0) \phi_{4}^{2}(0)+m_{38} \phi_{4}^{3}(0)+m_{39} \phi_{2}(0) \phi_{4}(0) \cdots \\
0 \\
0
\end{array}\right),
\end{aligned}
$$

with

$$
M_{1}=\left(\begin{array}{ccccc}
m_{11} & m_{12} & 0 & m_{14} & 0 \\
0 & m_{22} & 0 & m_{24} & 0 \\
m_{31} & m_{32} & m_{33} & m_{34} & 0 \\
0 & 0 & 0 & m_{44} & 0 \\
a_{51} & 0 & 0 & m_{54} & m_{55}
\end{array}\right) \text {, }
$$

$$
\begin{aligned}
& M_{2}=\left(\begin{array}{ccccc}
0 & 0 & 0 & 0 & 0 \\
0 & 0 & 0 & 0 & 0 \\
0 & 0 & n_{33} & 0 & 0 \\
0 & 0 & n_{43} & 0 & 0 \\
0 & 0 & 0 & 0 & 0
\end{array}\right), \\
& m_{15}=-\frac{\beta}{\left(1+\eta I_{*}\right)^{2}}
\end{aligned}
$$




$$
\begin{aligned}
& m_{16}=\frac{\beta \eta S_{*}}{\left(1+\eta I_{*}\right)^{3}}, \\
& m_{17}=\frac{\beta \eta}{\left(1+\eta I_{*}\right)^{3}}, \\
& m_{18}=-\frac{\beta \eta^{2} S_{*}}{\left(1+\eta I_{*}\right)^{4}}, \\
& m_{25}=-\sigma \beta, \\
& m_{35}=\frac{\beta}{\left(1+\eta I_{*}\right)^{2}}, \\
& m_{36}=-\frac{\beta \eta S_{*}}{\left(1+\eta I_{*}\right)^{3}}, \\
& m_{37}=-\frac{\beta \eta}{\left(1+\eta I_{*}\right)^{3}}, \\
& m_{39}=\sigma \beta \\
& m_{38}=\frac{\beta \eta^{2} S_{*}}{\left(1+\eta I_{*}\right)^{4}}, \\
& m
\end{aligned}
$$

According to the Riesz representation theorem, there exists a $5 \times 5$ matrix function $\eta(\theta, \varrho): \theta \in[-1,0] \rightarrow R^{5}$ such that

$$
L_{\varrho} \phi=\int_{-1}^{0} d \eta(\theta, \varrho) \phi(\theta), \quad \text { for } \phi \in C .
$$

In fact, choosing

$$
\eta(\theta, \varrho)=\left(\tau_{0}+\varrho\right)\left(A_{\max } \delta(\theta)+B_{\max } \delta(\theta+1)\right)
$$

and $\delta(\theta)$ is the Dirac delta function.

For $\phi \in C\left([-1,0], R^{5}\right)$, define

$$
\begin{aligned}
& A(\varrho) \phi= \begin{cases}\frac{d \phi(\theta)}{d \theta}, & -1 \leq \theta<0, \\
\int_{-1}^{0} d \eta(\theta, \varrho) \phi(\theta), & \theta=0,\end{cases} \\
& R(\varrho) \phi= \begin{cases}0, & -1 \leq \theta<0, \\
F(\varrho, \phi), & \theta=0 .\end{cases}
\end{aligned}
$$

Then system (21) can be transformed into the following operator equation:

$$
\dot{u}(t)=A(\varrho) u_{t}+R(\varrho) u_{t} .
$$

For $\varphi \in C^{1}\left([0,1],\left(R^{5}\right)^{*}\right)$, we further define the adjoint operator

$$
A^{*}(\varphi)= \begin{cases}-\frac{d \varphi(s)}{d s}, & 0<s \leq 1, \\ \int_{-1}^{0} d \eta^{T}(s, 0) \varphi(-s), & s=0\end{cases}
$$

and the bilinear inner product as follows:

$$
\begin{aligned}
\langle\varphi(s), \phi(\theta)\rangle= & \bar{\varphi}(0) \phi(0) \\
& -\int_{\theta=-1}^{0} \int_{\xi=0}^{\theta} \bar{\varphi}(\xi-\theta) d \eta(\theta) \phi(\xi) d \xi
\end{aligned}
$$

where $\eta(\theta)=\eta(\theta, 0)$.

Based on the discussion above, we know that $\pm i \omega_{0} \tau_{0}$ are eigenvalues of $A(0)$. Thus, they are also eigenvalues of $A^{*}$. Let $q(\theta)=\left(1, q_{2}, q_{3}, q_{4}, q_{5}\right)^{T} e^{i \tau_{0} \omega_{0} \theta}$ be the eigenvector of $A(0)$ corresponding to $+i \omega_{0} \tau_{0}$ and $q^{*}(s)=(1 / D)\left(1, q_{2}^{*}\right.$, $\left.q_{3}^{*}, q_{4}^{*}, q_{5}^{*}\right)^{T} e^{i \tau_{0} \omega_{0} s}$ be the eigenvectors of $A^{*}$ corresponding to $-i \tau_{0} \omega_{0}$. By direct computation, we can obtain

$$
\begin{aligned}
& q_{2}=\frac{m_{24}\left(i \omega_{0}-m_{11}\right)}{m_{12} m_{24}+m_{14}\left(i \omega_{0}-m_{22}\right)}, \\
& q_{3}=\frac{i \omega_{0}-m_{44}}{n_{43} e^{-i \tau_{0} \omega_{0}} q_{4},} \\
& q_{4}=\frac{i \omega_{0}-m_{22}}{m_{24}} q_{2}, \\
& q_{5}=\frac{m_{54}}{i \omega_{0}-m_{55}} q_{4}, \\
& q_{2}^{*}=-\frac{m_{12}+m_{32} q_{3}}{i \omega_{0}+m_{22}}, \\
& q_{3}^{*}=-\frac{i \omega_{0}+m_{11}}{m_{31}}, \\
& q_{4}^{*}=-\frac{i \omega_{0}+m_{33}+n_{33} e^{i \tau_{0} \omega_{0}}}{n_{43} e^{i \tau_{0} \omega_{0}}}, \\
& q_{5}^{*}=-\frac{m_{24} q_{2}^{*}+m_{34} q_{3}^{*}+\left(i \omega_{0}+m_{11}\right) q_{4}^{*}}{m_{54}}, \\
& \bar{D}=1+q_{2} \bar{q}_{2}^{*}+q_{3} \bar{q}_{3}^{*}+q_{4} \bar{q}_{4}^{*}+q_{5} \bar{q}_{5}^{*}\left(n_{33} \bar{q}_{3}^{*}+n_{43} \bar{q}_{4}^{*}\right) .
\end{aligned}
$$

Then we have $\left\langle q^{*}, q\right\rangle=1$ and $\left\langle q^{*}, \bar{q}\right\rangle=0$.

Next, we can obtain the coefficients which can determine the properties of the Hopf bifurcation at $\tau_{0}$ by following the algorithms given in [33] and using the computation process similar to those in [34-36]:

$$
\begin{aligned}
g_{20} & =\frac{2 \tau_{0}}{\bar{D}}\left[m_{15} q_{4}+m_{16} q_{4}^{2}+m_{25} \bar{q}_{2}^{*} q_{2} q_{4}+\bar{q}_{3}^{*}\left(m_{35} q_{4}\right.\right. \\
& \left.\left.+m_{36} q_{4}^{2}+m_{39} q_{2} q_{4}\right)\right], \\
g_{11} & =\frac{\tau_{0}}{\bar{D}}\left[m_{15}\left(q_{4}+\bar{q}_{4}\right)+2 m_{16} q_{4} \bar{q}_{4}+m_{25} \bar{q}_{2}^{*}\left(q_{2} \bar{q}_{4}\right.\right. \\
& \left.+\bar{q}_{2} q_{4}\right)+\bar{q}_{3}^{*}\left(m_{35}\left(q_{4}+\overline{q_{4}}\right)+2 m_{36} q_{4} \bar{q}_{4}\right. \\
& \left.\left.+m_{39}\left(q_{2} \bar{q}_{4}+\bar{q}_{2} q_{4}\right)\right)\right]
\end{aligned}
$$




$$
\begin{aligned}
g_{02} & =\frac{2 \tau_{0}}{\bar{D}}\left[m_{15} \bar{q}_{4}+m_{16} \bar{q}_{4}^{2}+m_{25} \bar{q}_{2}^{*} \bar{q}_{2} \bar{q}_{4}+\bar{q}_{3}^{*}\left(m_{35} \bar{q}_{4}\right.\right. \\
& \left.\left.+m_{36} \bar{q}_{4}^{2}+m_{39} \bar{q}_{2} \bar{q}_{4}\right)\right] \\
g_{21} & =\frac{2 \tau_{0}}{\bar{D}}\left[m _ { 1 5 } \left(\frac{1}{2} W_{20}^{(4)}(0)+W_{11}^{(4)}(0)+\frac{1}{2} W_{20}^{(1)}(0)\right.\right. \\
& \left.+W_{11}^{(1)}(0) \bar{q}_{4}\right)+m_{16}\left(W_{20}^{(4)}(0) \bar{q}_{4}+2 W_{11}^{(4)}(0) q_{4}\right) \\
& +m_{17}\left(q_{4}^{2}+2 q_{4} \bar{q}_{4}\right)+3 m_{18} q_{4}^{2} \bar{q}_{4}+m_{25} \bar{q}_{2}^{*}\left(\frac{1}{2}\right. \\
& +W_{20}^{(2)}(0) \bar{q}_{4}+W_{11}^{(2)}(0) q_{4}+\frac{1}{2} W_{20}^{(4)}(0) \bar{q}_{2} \\
& \left.+W_{11}^{(4)}(0) q_{2}\right)+\bar{q}_{3}^{*}\left(m _ { 3 5 } \left(\frac{1}{2} W_{20}^{(4)}(0)+W_{11}^{(4)}(0)\right.\right. \\
& \left.\left.\left.+W_{11}^{(4)}(0) q_{2}\right)\right)\right], \\
& \left.+\frac{1}{2} W_{20}^{(1)}(0)+W_{11}^{(1)}(0) \bar{q}_{4}\right)+m_{36}\left(W_{20}^{(4)}(0) \bar{q}_{4}\right. \\
& \left.+2 W_{11}^{(4)}(0) q_{4}\right)+m_{37}\left(q_{4}^{2}+2 q_{4} \bar{q}_{4}\right)+3 m_{38} q_{4}^{2} \bar{q}_{4} \\
& +m_{39}\left(\frac{1}{2} W_{20}^{(2)}(0) \bar{q}_{4}+W_{11}^{(2)}(0) q_{4}+\frac{1}{2} W_{20}^{(4)}(0) \bar{q}_{2}\right.
\end{aligned}
$$

with

$$
\begin{aligned}
& W_{20}(\theta) \\
& =\frac{i g_{20} \rho(0)}{\tau_{0} \omega_{0}} e^{i \tau_{0} \omega_{0} \theta}+\frac{i \bar{g}_{02} \bar{\rho}(0)}{3 \tau_{0} \omega_{0}} e^{-i \tau_{0} \omega_{0} \theta}+E_{1} e^{2 i \tau_{0} \omega_{0} \theta} \\
& W_{11}(\theta)=-\frac{i g_{11} \rho(0)}{\tau_{0} \omega_{0}} e^{i \tau_{0} \omega_{0} \theta}+\frac{i \bar{g}_{11} \bar{\rho}(0)}{\tau_{0} \omega_{0}} e^{-i \tau_{0} \omega_{0} \theta}+E_{2}, \\
& E_{1} \\
& =2\left(\begin{array}{ccccc}
m_{11}^{\prime} & -m_{12} & 0 & -m_{14} & 0 \\
0 & m_{22}^{\prime} & 0 & -m_{24} & 0 \\
-m_{31} & -m_{32} & m_{33}^{\prime} & -m_{34} & 0 \\
0 & 0 & -n_{43} e^{-2 i \tau_{0} \omega_{0}} & m_{44}^{\prime} & 0 \\
0 & 0 & 0 & -m_{54} & m_{55}^{\prime}
\end{array}\right)^{-1} \\
& \times\left(\begin{array}{c}
E_{1}^{(1)} \\
E_{1}^{(2)} \\
E_{1}^{(3)} \\
0 \\
0
\end{array}\right),
\end{aligned}
$$

$E_{2}$ $=-\left(\begin{array}{ccccc}m_{11} & m_{12} & 0 & m_{14} & 0 \\ 0 & m_{22} & 0 & m_{24} & 0 \\ m_{31} & m_{32} & m_{33}+n_{33} & m_{34} & 0 \\ 0 & 0 & n_{43} & m_{44} & 0 \\ 0 & 0 & 0 & m_{54} & m_{55}\end{array}\right)^{-1}$ $\times\left(\begin{array}{c}E_{2}^{(1)} \\ E_{2}^{(2)} \\ E_{2}^{(3)} \\ 0 \\ 0\end{array}\right)$

where

$$
\begin{aligned}
& m_{11}^{\prime}=2 i \omega_{0}-m_{11}, \\
& m_{22}^{\prime}=2 i \omega_{0}-m_{22}, \\
& m_{33}^{\prime}=2 i \omega_{0}-m_{33}-n_{33} e^{-2 i \tau_{0} \omega_{0},} \\
& m_{44}^{\prime}=2 i \omega_{0}-m_{44}, \\
& m_{55}^{\prime}=2 i \omega_{0}-m_{55}, \\
& E_{1}^{(1)}=m_{15} q_{4}+m_{16} q_{4}^{2}, \\
& E_{1}^{(2)}=m_{25} q_{2} q_{4}, \\
& E_{1}^{(3)}=m_{35} q_{4}+m_{36} q_{4}^{2}+m_{39} q_{2} q_{4}, \\
& E_{2}^{(1)}=m_{15}\left(q_{4}+\bar{q}_{4}\right)+2 m_{16} q_{4} \bar{q}_{4}, \\
& E_{2}^{(2)}=m_{25}\left(q_{2} \bar{q}_{4}+\bar{q}_{2} q_{4}\right), \\
& E_{2}^{(3)}=m_{35}\left(q_{4}+\bar{q}_{4}\right)+2 m_{36} q_{4} \bar{q}_{4}, \\
& \quad+m_{39}\left(q_{2} \bar{q}_{4}+\bar{q}_{2} q_{4}\right) .
\end{aligned}
$$

Then, one can obtain

$$
\begin{aligned}
C_{1}(0) & =\frac{i}{2 \tau_{0} \omega_{0}}\left(g_{11} g_{20}-2\left|g_{11}\right|^{2}-\frac{\left|g_{02}\right|^{2}}{3}\right)+\frac{g_{21}}{2} \\
\mu_{2} & =-\frac{\operatorname{Re}\left\{C_{1}(0)\right\}}{\operatorname{Re}\left\{\lambda^{\prime}\left(\tau_{0}\right)\right\}}, \\
\beta_{2} & =2 \operatorname{Re}\left\{C_{1}(0)\right\}, \\
T_{2} & =-\frac{\operatorname{Im}\left\{C_{1}(0)\right\}+\mu_{2} \operatorname{Im}\left\{\lambda^{\prime}\left(\tau_{0}\right)\right\}}{\tau_{0} \omega_{0}} .
\end{aligned}
$$

In conclusion, we have the following results.

Theorem 2. For system (2), $\mu_{2}$ determines the direction of the Hopf bifurcation: if $\mu_{2}>0\left(\mu_{2}<0\right)$, then the Hopf bifurcation 
is supercritical (subcritical); $\beta_{2}$ determines the stability of the bifurcating periodic solution: the bifurcating periodic solutions are stable (unstable) if $\beta_{2}<0\left(\beta_{2}>0\right) ; T_{2}$ determines the period of the bifurcating periodic solutions: the period increases (decreases) if $T_{2}>0\left(T_{2}<0\right)$.

\section{Numerical Simulation}

In this section, some numerical simulations are carried out for qualitative analysis by using Matlab software package. By extracting some values from [29] and considering the conditions for the existence of the Hopf bifurcation, we choose a set of parameters as follows: $A=100, p=0.5$, $\alpha=0.0001, \beta=0.003, \gamma=0.0001, \delta=0.4, \eta=1$, $\sigma=0.05 \omega=0.02$, and $\mu=0.001$. Then, we can get the following specific case of system (2):

$$
\begin{aligned}
\frac{d S(t)}{d t}= & 50-\frac{0.003 S(t) I(t)}{1+I(t)}-0.001 S(t) \\
& +0.0001 V(t), \\
\frac{d V(t)}{d t}= & 50-1.5000 e-004 V(t) I(t) \\
& -0.0011 V(t), \\
\frac{d E(t)}{d t}= & \frac{0.003 S(t) I(t)}{1+I(t)}+1.5000 e-004 V(t) I(t) \\
& -0.001 E(t)-0.02 E(t-\tau), \\
\frac{d I(t)}{d t}= & 0.02 E(t-\tau)-0.4011 I(t), \\
\frac{d R(t)}{d t}= & 0.4 I(t)-0.0001 R(t) .
\end{aligned}
$$

By some computations, we can obtain the following equation with respect to $I$ :

$$
\begin{aligned}
& 5.0539 e-009 I^{2}-1.0117 e-006 I-4.7907 e-006 \\
& \quad=0 .
\end{aligned}
$$

It follows that system (35) has a unique positive equilibrium $P_{*}(12723,1571.3,4107.5,204.8103,81924)$ and we can verify that $P_{*}(12723,1571.3,4107.5,204.8103,81924)$ is locally asymptotically stable when $\tau=0$. Further, we have $\omega_{0}=0.5508$ and $\tau_{0}=69.6986$. According to Theorem 1 , it can be concluded that $P_{*}(12723,1571.3,4107.5,204.8103,81924)$ is locally asymptotically stable when $\tau \in\left[0, \tau_{0}=69.6986\right)$. This property can be shown as in Figures 1 and 2. However, a Hopf bifurcation will occur and a family of periodic solutions bifurcate from $P_{*}(12723,1571.3,4107.5,204.8103,81924)$ when the value of $\tau$ passes through the Hopf bifurcation value $\tau_{0}$, which can be illustrated by Figures 3 and 4.

In addition, we obtain $C_{1}(0)=-4.3990+2.9057 i, \lambda^{\prime}\left(\tau_{0}\right)=$ $0.7014-0.0212 i$ by some complicate computations. Thus, we get $\mu_{2}=7.8776>0, \beta_{2}=-8.798<0$, and $T_{2}=$ $-0.0713<0$ based on (34). It follows from Theorem 2 that

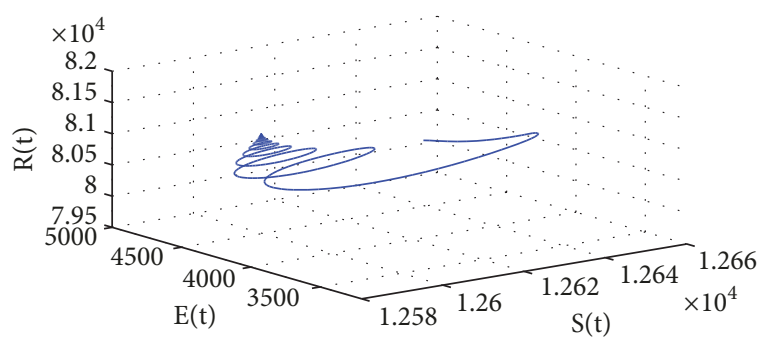

FIgURE 1: Dynamic behavior of system (35): projection on S-E-R with $\tau=65.85<\tau_{0}$.

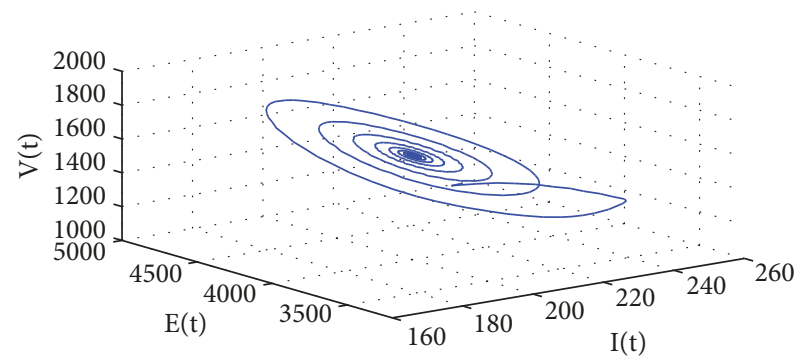

FIGURE 2: Dynamic behavior of system (35): projection on V-E-I with $\tau=65.85<\tau_{0}$.

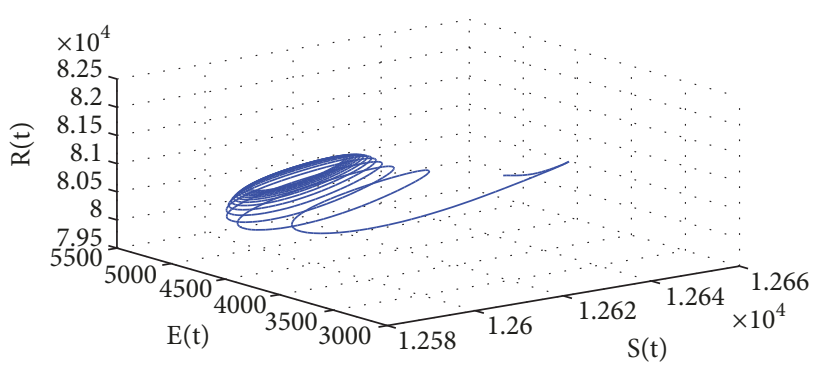

Figure 3: Dynamic behavior of system (35): projection on S-E-R with $\tau=76.65>\tau_{0}$.

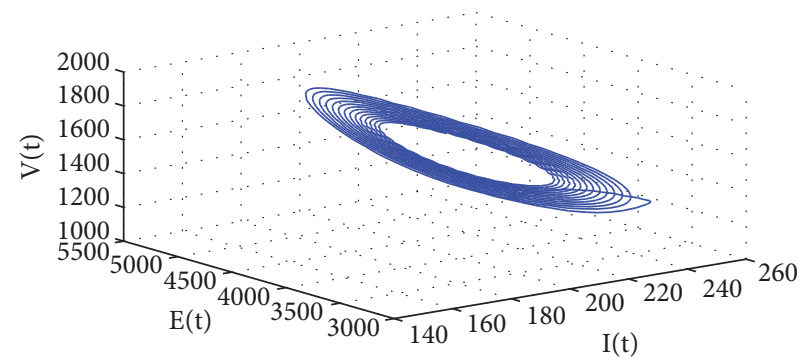

Figure 4: Dynamic behavior of system (35): projection on V-E-I with $\tau=76.65>\tau_{0}$.

the Hopf bifurcation is supercritical and the bifurcating periodic solutions are stable and decrease. Since the bifurcating periodic solutions are stable, then the five classes of hosts in system (35) may coexist in an oscillatory mode from the view of the biological point, which is not welcome in networks. 


\section{Conclusions}

In this study, the dynamical behaviors of a delayed SVEIR worm propagation model with saturated incidence are discussed based on the work in literature [29]. The dynamical behaviors of the model are investigated from the point of view of local stability and Hopf bifurcation both analytically and numerically. The threshold of the time delay $\tau_{0}$ at which the model causes a Hopf bifurcation is obtained by using eigenvalue method. We found that characteristics of the propagation of worms in the model can be predicted and controlled when the value of delay is suitably small $(\tau \in$ $\left.\left[0, \tau_{0}\right)\right)$. However, propagation of the worms in the model will be out of control once the value of the time delay is above the threshold value $\tau_{0}$. Accordingly, we can know that the propagation of worms in the model can be controlled by postponing occurrence of the Hopf bifurcation. Moreover, the properties of the Hopf bifurcation are investigated by applying the normal form theory and the center manifold theorem. Numerical simulations are also presented in order to testify our obtained theoretical results.

\section{Parameters of the Model and Their Meanings}
A: Recruitment rate of the susceptible host
$p$ : Vaccinated rate of the susceptible host
$\beta$ : Infection rate of the susceptible host
$\sigma \beta$ : Infection rate of the vaccinated host
$\eta$ : Efficient measuring the inhibitory effect
$\mu$ : Natural death rate of all the hosts
$\alpha$ : Death rate of the infectious host due to worm attack
$\delta$ : Recovery rate of the infectious hosts
$\varpi$ : Rate of the exposed hosts that become infectious
$\gamma$ : Rate of the vaccinated hosts that become susceptible.

\section{Data Availability}

All data can be accessed in the numerical simulation section of this article.

\section{Conflicts of Interest}

The authors declare that there are no conflicts of interest regarding the publication of this paper.

\section{Acknowledgments}

This work was supported by Project of Support Program for Excellent Youth Talent in Colleges and Universities of Anhui Province (no. gxyqZD2018044) and Anhui Provincial Natural Science Foundation (no. 1608085QF145 and no. 1608085QF151).

\section{References}

[1] D. Moore, V. Paxson, S. Savage, C. Shannon, S. Staniford, and N. Weaver, "Inside the slammer worm," IEEE Security \& Privacy, vol. 1, no. 4, pp. 33-39, 2003.
[2] W. XIA, Z.-H. LI, Z.-Q. CHEN, and Z.-Z. YUAN, "Commwarrior worm propagation model for smart phone networks," Journal of China Universities of Posts and Telecommunications, vol. 15, no. 2, pp. 60-66, 2008.

[3] M. La Polla, F. Martinelli, and D. Sgandurra, "A survey on security for mobile devices," IEEE Communications Surveys \& Tutorials, vol. 15, no. 1, pp. 446-471, 2013.

[4] J. Scharr, "New WiFi Worm can Spread Like an Airborne Disease," https://www.yahoo.com/tech/new-wifi-worm-canspread-like-an-airborne-disease-78496514830.html.

[5] P. Szor, The Art of Computer Virus Research and Defense, Addison-Wesley, 2005.

[6] C. C. Zou, D. Towsley, and W. Gong, "On the performance of Internet worm scanning strategies," Performance Evaluation, vol. 63, no. 7, pp. 700-723, 2006.

[7] O. A. Toutonji, S.-M. Yoo, and M. Park, "Stability analysis of VEISV propagation modeling for network worm attack," Applied Mathematical Modelling: Simulation and Computation for Engineering and Environmental Systems, vol. 36, no. 6, pp. 2751-2761, 2012.

[8] W. O. Kermack and A. G. McKendrick, "A contribution to the mathematical theory of epidemics," Proceedings of the Royal Society A Mathematical, Physical and Engineering Sciences, vol. 115 , no. 772, pp. 700-721, 1927.

[9] J. Kim, S. Radhakrishnan, and S. Dhall, "Measurement and analysis of worm propagation on Internet network topology," in Proceedings of the 13th International Conference on Computer Communications and Networks, pp. 495-500, Chicago, IL, USA.

[10] X. M. Wang and Y. S. Li, "An improved SIR model for analyzing the dynamics of worm propagation in wireless sensor networks," Journal of Electronics, vol. 18, no. 1, pp. 8-12, 2009.

[11] B. K. Mishra and A. K. Singh, "Global stability of worms in computer network," Applications and Applied Mathematics. An International Journal, vol. 5, no. 10, pp. 1511-1528, 2010.

[12] L. Feng, L. Song, Q. Zhao, and H. Wang, "Modeling and stability analysis of worm propagation in wireless sensor network," Mathematical Problems in Engineering, Article ID 129598, Art. ID 129598, 8 pages, 2015.

[13] B. K. Mishra and A. Prajapati, "Cyber Warfare : Worms' Transmission Model," International Journal of Advanced Science and Technology, vol. 63, pp. 83-94, 2014.

[14] N. Keshri and B. K. Mishra, "Two time-delay dynamic model on the transmission of malicious signals in wireless sensor network," Chaos, Solitons \& Fractals, vol. 68, pp. 151-158, 2014.

[15] B. K. Mishra and S. K. Pandey, "Dynamic model of worms with vertical transmission in computer network," Applied Mathematics and Computation, vol. 217, no. 21, pp. 8438-8446, 2011.

[16] R. P. Ojha, G. Sanyal, P. K. Srivastava, and K. Sharma, "Design and analysis of modified SIQRS model for performance study of wireless sensor network," Scalable Computing: Practice and Experience, vol. 18, no. 3, pp. 229-241, 2017.

[17] Y. Yao, X.-w. Xie, H. Guo, G. Yu, F.-X. Gao, and X.-j. Tong, "Hopf bifurcation in an Internet worm propagation model with time delay in quarantine," Mathematical and Computer Modelling, vol. 57, no. 11-12, pp. 2635-2646, 2013.

[18] X. Xiao, P. Fu, C. Dou, Q. Li, G. Hu, and S. Xia, "Design and analysis of SEIQR worm propagation model in mobile internet. Communications in Nonlinear Science $£$ Numerical Simulation," Communications in Nonlinear Science and Numerical Simulation, vol. 43, pp. 341-350, 2017. 
[19] Y. Yao, Q. Fu, C. Sheng, and W. Yang, "Modeling and hopf bifurcation analysis of benign worms with quarantine strategy," Lecture Notes in Computer Science (including subseries Lecture Notes in Artificial Intelligence and Lecture Notes in Bioinformatics): Preface, vol. 10581, pp. 320-336, 2017.

[20] F. Wang, Y. Yang, D. Zhao, and Y. Zhang, "A worm defending model with partial immunization and its stability analysis," Journal of Communications, vol. 10, no. 4, pp. 276-283, 2015.

[21] B. K. Mishra and S. K. Pandey, "Dynamic model of worm propagation in computer network," Applied Mathematical Modelling: Simulation and Computation for Engineering and Environmental Systems, vol. 38, no. 7-8, pp. 2173-2179, 2014.

[22] C. H. Nwokoye and I. I. Umeh, "The SEIQR-V Model: On a More Accurate Analytical Characterization of Malicious Threat Defense," International Journal of Information Technology and Computer Science, vol. 9, no. 12, pp. 28-37, 2017.

[23] B. K. Mishra and N. Keshri, "Mathematical model on the transmission of worms in wireless sensor network," Applied Mathematical Modelling: Simulation and Computation for Engineering and Environmental Systems, vol. 37, no. 6, pp. 4103-4111, 2013.

[24] A. Singh, A. K. Awasthi, K. Singh, and P. K. Srivastava, "Modeling and Analysis of Worm Propagation in Wireless Sensor Networks," Wireless Personal Communications, pp. 1-17, 2017.

[25] M. Kumar, B. K. Mishra, and T. C. Panda, "Effect of quarantine ú vaccination on infectious nodes in computer network," in Proceedings of the Effect of quarantine $£$ vaccination on infectious nodes in computer network, p. 92, 2015.

[26] R. K. Upadhyay, S. Kumari, and A. K. Misra, "Modeling the virus dynamics in computer network with SVEIR model and nonlinear incident rate," Applied Mathematics and Computation, vol. 54, no. 1-2, pp. 485-509, 2017.

[27] C. Gan, X. Yang, W. Liu, Q. Zhu, and X. Zhang, "An epidemic model of computer viruses with vaccination and generalized nonlinear incidence rate," Applied Mathematics and Computation, vol. 222, pp. 265-274, 2013.

[28] C. Gan, X. Yang, W. Liu, and Q. Zhu, "A propagation model of computer virus with nonlinear vaccination probability," Communications in Nonlinear Science and Numerical Simulation, vol. 19, no. 1, pp. 92-100, 2014.

[29] F. Wang, W. Huang, Y. Shen, and C. Wang, "Analysis of SVEIR worm attack model with saturated incidence and partial immunization," Journal of Communications and Information Networks, vol. 1, no. 4, pp. 105-115, 2016.

[30] J. Ren, X. Yang, L.-X. Yang, Y. Xu, and F. Yang, "A delayed computer virus propagation model and its dynamics," Chaos, Solitons \& Fractals, vol. 45, no. 1, pp. 74-79, 2012.

[31] Y. B. Kafai, "Understanding virtual epidemics: children's folk conceptions of a computer virus," Journal of Science Education and Technology, vol. 17, no. 6, pp. 523-529, 2008.

[32] T. Zhang, H. Jiang, and Z. Teng, "On the distribution of the roots of a fifth degree exponential polynomial with application to a delayed neural network model," Neurocomputing, vol. 72, no. 46, pp. 1098-1104, 2009.

[33] B. D. Hassard, N. D. Kazarinoff, and Y.-H. Wan, Theory and Applications of Hopf Bifurcation, Cambridge University Press, 1981.

[34] X.-Y. Meng, H.-F. Huo, X.-B. Zhang, and H. Xiang, "Stability and Hopf bifurcation in a three-species system with feedback delays," Nonlinear Dynamics, vol. 64, no. 4, pp. 349-364, 2011.
[35] D. Jana, R. Agrawal, and R. K. Upadhyay, "Top-predator interference and gestation delay as determinants of the dynamics of a realistic model food chain," Chaos, Solitons \& Fractals, vol. 69, pp. 50-63, 2014.

[36] R. K. Upadhyay and R. Agrawal, "Dynamics and responses of a predator-prey system with competitive interference and time delay," Nonlinear Dynamics, vol. 83, no. 1-2, pp. 821-837, 2016. 


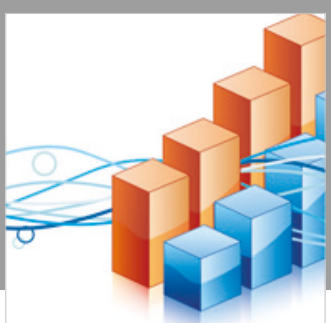

Advances in

Operations Research

\section{-n-m}
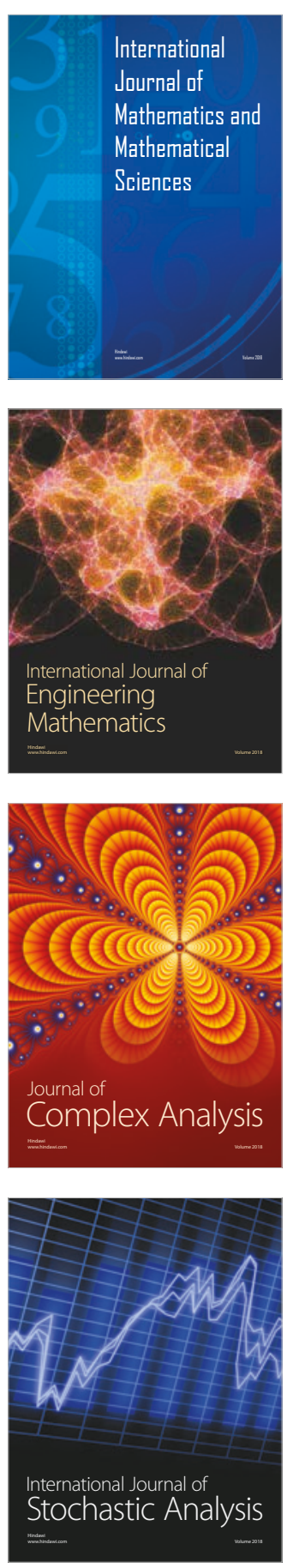
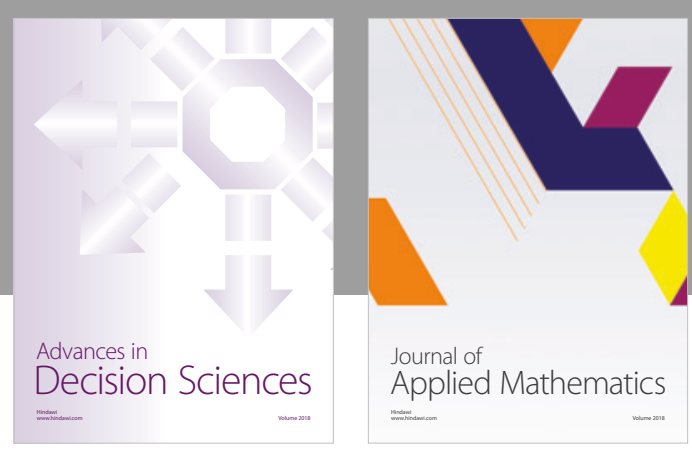

Journal of

Applied Mathematics
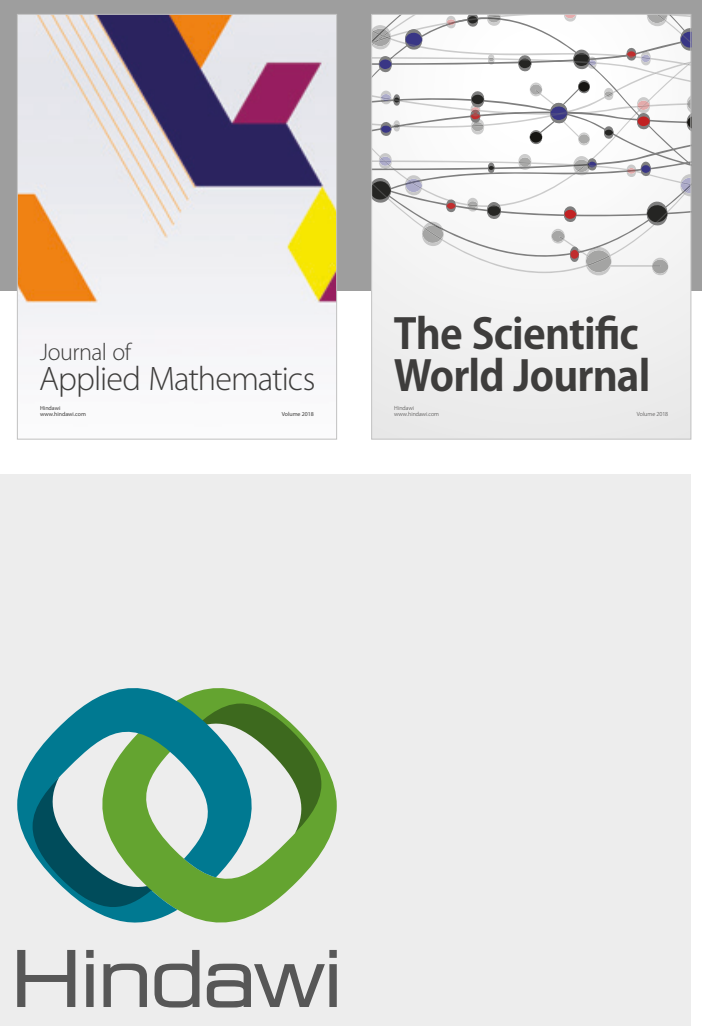

Submit your manuscripts at

www.hindawi.com

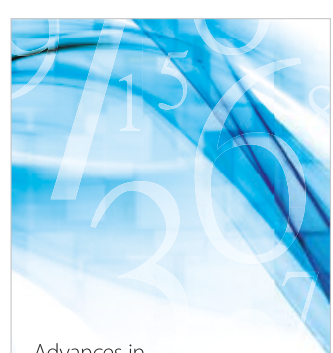

Advances in
Numerical Analysis
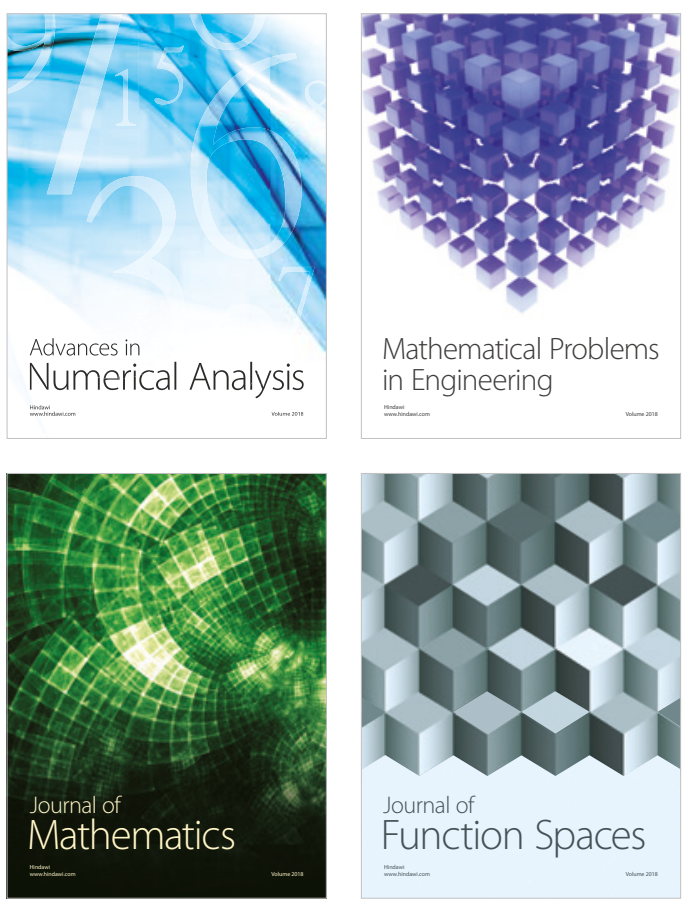

Mathematical Problems in Engineering

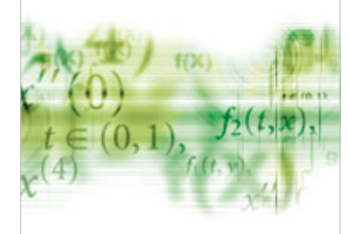

International Journal of

Differential Equations

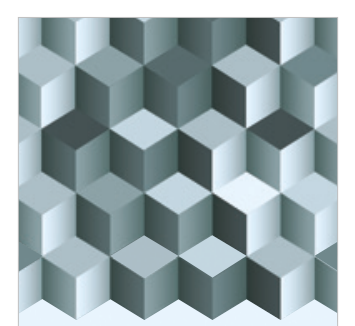

Journal of

Function Spaces

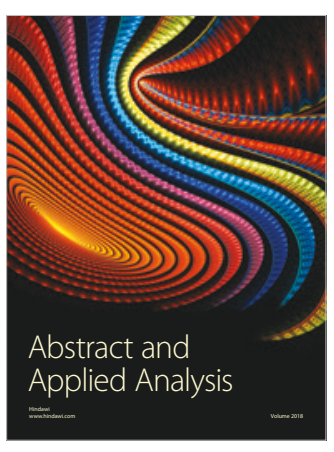

The Scientific

World Journal

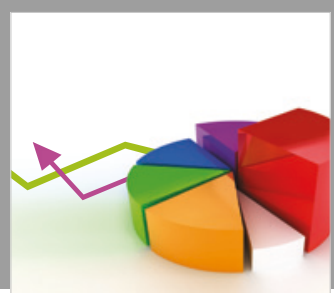

Journal of

Probability and Statistics
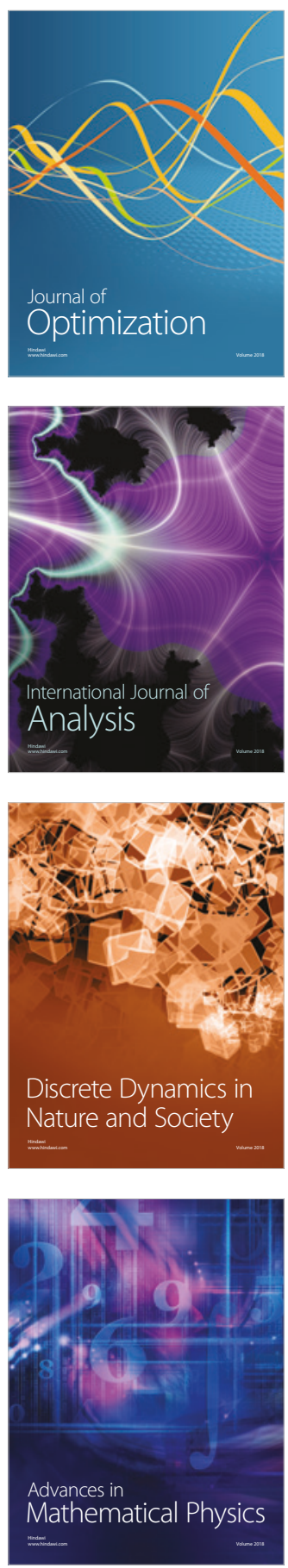